\title{
Einleitung Teil III
}

\section{Ulrich Reimer}

\section{Linked (Open) Data: Mehrwerte für Bibliotheken und Archive}

Unter Linked Data versteht man die technologischen Ansätze eines World Wide Web der Daten - im Unterschied zum ursprünglichen Web der Dokumente. So werden Datenbestände über das Web zugreifbar und können sich darüber hinaus gegenseitig referenzieren. Daten, die an einem Ort gespeichert sind, lassen sich durch Verlinkung darauf in einen neuen Datenbestand einbinden und müssen nicht erneut erstellt werden. Unnötiger Zusatzaufwand, Redundanz und daraus entstehende Inkonsistenzen werden so vermieden. Werden Linked Data Datenbestände als Open Data bereitgestellt, spricht man von Linked Open Data (LOD).

Bibliotheken und mehr noch Archive führen ihre Datenbestände traditionell lokal, doch werden die neuen Möglichkeiten, die sich mit Linked Data ergeben, zunehmend genutzt. Für Bibliotheken bedeutet dies z.B. die leichtere Wiederverwendung von Erschliessungsinformationen anderer Bibliotheken, für Archive ermöglicht der Ansatz von Linked Data die Bereitstellung der Archivdaten auf dem Web, so dass sie nicht nur einem breiteren Benutzerkreis zugänglich, sondern insbesondere durch Programme abfragbar und auswertbar werden.

Die Verknüpfung von Daten über Datenbestände hinweg kann nur gelingen, wenn es eine terminologische Kontrolle gibt, gleiche Dinge also gleich benannt und beschrieben werden. Gemeinsame Vokabulare werden durch Thesauri bzw. Ontologien bereitgestellt, welche als terminologische Schemata für Link Data Datenbestände fungieren und so semantische Interoperabilität ermöglichen. Linked Data basiert damit auf den Konzepten des Semantic Web.

Die beiden im Folgenden vorgestellten Beiträge zeigen die Anwendungsmöglichkeiten von Linked (Open) Data für Bibliotheken und Archive auf und legen dar, welche Überlegungen in einer konkreten Umsetzung notwendig sind und welches Vorgehen jeweils zu empfehlen ist.

\section{L'enrichissement automatique de l'indexation dans le réseau Renouvaud} Traditionell erfolgt die Sacherschliessung in Bibliotheken mittels Schlagwörter aus einem kontrollierten Vokabular. Dies geschieht auf manuelle Weise und verlangt eine fundierte Expertise seitens der Sacherschliesser. Es lässt sich so eine hohe Konsistenz und Qualität der Indexierung erreichen, doch bedeutet dies auch einen hohen Zeitaufwand und ist somit sehr kostspielig. Vor allem vor dem Hintergrund der stetig wachsenden Menge an zu indexierendem Material ist die manuelle Indexierung kaum noch 
praktikabel. Insbesondere stellt sich die Frage, ob jede Bibliothek ihre Bestände selber indexieren muss oder stattdessen auf eine schon bestehende Indexierung durch andere Bibliotheken zurückgreift, um so den zu leistenden Aufwand zu reduzieren und die Qualität zu verbessern.

MICHAEL HERTIG, Bibliothèque cantonale et universitaire Lausanne, untersucht in seiner Arbeit die Schwierigkeiten, welche entstehen, wenn ein Bibliothekskatalog um Erschliessungsinformation von anderen Bibliotheken angereichert wird. Dies kann prinzipiell auf zwei Arten erfolgen: durch Importieren in den Katalog oder durch das Hinzufügen von Verweisen auf die externen Ressourcen. Das zweite Vorgehen folgt dem Paradigma von Linked Data, wo Daten an ihrem Ursprungsort bleiben und lediglich miteinander verknüpft werden.

In beiden Fällen bedeutet die Integration unterschiedlich entstandener Erschliessungsinformation, dass der resultierende Katalog Angaben auf der Basis unterschiedlicher Vokabulare enthält - das von der Bibliothek selber verwendete Vokabular sowie die Vokabulare der importierten Erschliessungsinformation. Dies kann auch implizieren, dass Indexterme aus verschiedenen Sprachen stammen. Betroffene Ressourcen sind dann nicht mehr nur mittels eines Vokabulars auffindbar und verlangen vom Benutzer einen höheren Aufwand bei der Suche.

Michael Hertig erläutert in seiner Arbeit die Vor- und Nachteile der unterschiedlichen Vorgehensweisen und illustriert diese, indem er jeweils den Bezug zur konkreten Situation des Bibliotheksverbunds Waadt Renouvaud herstellt.

\section{Archival Linked (Open) Data}

Der schon seit einiger Zeit veröffentlichte, internationale Verzeichnisstandard für Archive Records in Context (RiC) verändert die Sichtweise auf Archive als isolierte Bestände hin zu vernetzten, maschinenlesbaren Beständen. Auch wenn die Weiterentwicklung des Standards und dessen Aufnahme durch Archive eher langsam verläuft, experimentieren mittlerweile einige bedeutende Archive mit diesem neuen Standard. Er basiert auf den Technologien des Semantic Web sowie von Linked Open Data (LOD) - den aktuellen Standards für semantische Interoperabilität. Die Öffnung von Archiven und ihre Transformation in Linked Data Datenbestände erschliessen ein grosses Potenzial für die Nutzung und Verbreitung des kulturellen Erbes.

Die Master-Arbeit von FABIAN WÜRTZ, Schweizerisches Sozialarchiv, die in voller Länge wiedergegeben ist, geht auf die spannenden Entwicklungen im Kontext von RiC ein. Nach Einführung der Grundlagen und einer Übersicht über die von einigen ausgewählten Archiven verwendeten Ansätze zur Erstellung einer LOD-Version stellt der Autor ein Vorgehensmodell für die Umwandlung eines Archivs in einen LOD-Datenbestand vor. Wie dies in einem konkreten Fall aussehen könnte, wird am Beispiel des Schweizerischen Sozialarchivs aufgezeigt. 
Das Vorgehensmodell ist speziell darauf ausgerichtet, eine hohe Datenqualität der resultierenden LOD-Version eines Archivs zu erzielen. Hierfür sind unterschiedliche Aspekte zu beachten, darunter die folgenden:

- Es ist ein geeignetes, LOD-fähiges Datenmodell für die Archivstruktur zu wählen.

- Die Bereitstellung der Metadaten eines Archivs als Open Data kann urheberrechtliche Aspekte implizieren. Fabian Würtz schlägt vor, die Metadaten mit Lizenzen zu versehen, um den späteren Benutzern eine möglichst hohe Rechtssicherheit zu bieten.

- Um die (Meta-)Daten des Archivs nicht nur innerhalb des Archivs, sondern auch mit externen Ressourcen zu verknüpfen, müssen sie terminologisch anbindungsfähig sein. Dazu ist das entsprechende Vokabular in Form eines oder mehrerer Thesauri bzw. Ontologien auszuwählen.

- Ein weiterer Aspekt ist die Anreicherung mit Metadaten zu den zu beschreibenden Archivobjekten sowie zu den schon bestehenden Metadaten (welche dann Meta-Metadaten wären). Zum Beispiel wäre die Angabe des Erstellers eines Objekts ein Metadatum, Angaben über den Ersteller wären dann Metadaten über das Metadatum «Ersteller». Die daraus resultierende Metadatenstruktur kann recht komplex werden und kann einiges an Aufwand bedeuten, um sie im Linked Data Format darzustellen.

- Veränderungen von Metadaten sollten über Versionierungen erfolgen, damit Auswertungen über den Archivbestand auch zu späteren Zeitpunkten reproduzierbar bleiben.

Bibliotheken sind bzgl. der Nutzung von LOD weiter fortgeschritten als Archive, weshalb Fabian Würtz empfiehlt, dass sich die Archivwelt mit Bibliotheken und verwandten Fachgebieten wie z.B. der Digitalisierung in Museen austauscht, um von ihren Erfahrungen zu profitieren.

\section{Wissensmanagement in einer sich stetig verändernden Organisation}

Wissensmanagement befasst sich mit der systematischen Bewahrung, Weiterentwicklung und Nutzung der Ressource Wissen - die wichtigste Ressource für den Erfolg einer Organisation. Die dabei zu bewältigenden Herausforderungen können je nach Organisation sehr unterschiedlich sein. Deshalb gestalten sich die implementierten Wissensmanagement-Massnahmen trotz bestehender Best Practices in jeder Organisation recht unterschiedlich.

Die Arbeit von SIMONA GENERELLI, Schweizer Archiv der Darstellenden Künste SAPA, befasst sich mit dem Wissensmanagement in der Direktion für Entwicklung und Zusammenarbeit (DEZA) der Bundesverwaltung. Die spezielle 
Herausforderung dort liegt in der häufigen Rotation der Mitarbeitenden zwischen den etwa vierzig Kooperationsbüros weltweit sowie der periodischen Zuweisung von Mitarbeitenden zu neuen Funktionen. So besteht die Gefahr, dass die erworbenen Kompetenzen und das Wissen einer Person nach dem Wechsel in eine neue Funktion für die Organisation verloren gehen und wieder neu erworben werden müssen. Ferner leidet durch die häufige Veränderung der Überblick, welche Person in der Organisation welche Expertise besitzt. Die DEZA weiss somit nur bedingt, über welches Wissen sie eigentlich verfügt. Neben dem implizitem, also dem an eine Person gebundenen Wissen, gilt es, das explizite Wissen in Form von Dokumenten aller Art zu managen.

Vor diesem Hintergrund untersucht Simona Generelli in ihrer Arbeit den Stand des Wissensmanagements in der DEZA, u.a. welche Instrumente für welchen Zweck eingesetzt werden und wo eventuell Verbesserungspotenzial besteht. Dazu setzt sie das von Gilbert Probst entwickelte Modell der Wissensbausteine ein, welches eine systematische Abdeckung aller Aspekte ermöglicht. Die Autorin analysiert für jeden Wissensbaustein relevante Hilfsmittel technischer Art (z.B. gemeinsame Dokumentablagen auf dem Intranet oder Wissenslandkarten für das leichtere Auffinden von Wissensressourcen), organisatorischer Art (z.B. für die Förderung des informellen Wissensaustauschs) sowie die Einstellung der Mitarbeitenden bzgl. Wissensmanagement, insbesondere ihre Motivation, die bestehenden Wissensmanagement-Werkzeuge tatsächlich anzuwenden.

Die Arbeit illustriert eindrucksvoll, vor welchen Herausforderungen Wissensmanagement in einer dynamischen Organisation stehen kann und wie sich diese adressieren lassen. 